\title{
Transynaptic Action of Botulinum Neurotoxin Type A at Central Cholinergic Boutons
}

\author{
Matteo Caleo, ${ }^{1,3 \star}$ Matteo Spinelli, ${ }^{1 \star}$ Francesca Colosimo, ${ }^{1}$ Ivica Matak, ${ }^{2}$ Ornella Rossetto, ${ }^{3}$ Zdravko Lackovic, ${ }^{2}$ \\ and Laura Restani ${ }^{1}$ \\ ${ }^{1}$ CNR Neuroscience Institute, Pisa 56124, Italy, ${ }^{2}$ Laboratory of Molecular Neuropharmacology, Department of Pharmacology, University of Zagreb School of \\ Medicine, 10000 Zagreb, Croatia, and ${ }^{3}$ Department of Biomedical Sciences, University of Padua, 35122 Padova, Italy
}

Botulinum neurotoxin Type A (BoNT/A) is an effective treatment for several movement disorders, including spasticity and dystonia. BoNT/A acts by cleaving synaptosomal-associated protein of $25 \mathrm{kDa}$ (SNAP-25) at the neuromuscular junction, thus blocking synaptic transmission and weakening overactive muscles. However, not all the therapeutic benefits of the neurotoxin are explained by peripheral neuroparalysis, suggesting an action of BoNT/A on central circuits. Currently, the specific targets of BoNT/A central activity remain unclear. Here, we show that catalytically active BoNT/A is transported to the facial nucleus (FN) after injection into the nasolabial musculature of rats and mice. BoNT/A-mediated cleavage of SNAP-25 in the FN is prevented by intracerebroventricular delivery of antitoxin antibodies, demonstrating that BoNT/A physically leaves the motoneurons to enter second-order neurons. Analysis of intoxicated terminals within the FN shows that BoNT/A is transcytosed preferentially into cholinergic synapses. The cholinergic boutons containing cleaved SNAP-25 are associated with a larger size, suggesting impaired neuroexocytosis. Together, the present findings indicate a previously unrecognized source of reduced motoneuron drive after BoNT/A via blockade of central, excitatory cholinergic inputs. These data highlight the ability of BoNT/A to selectively target and modulate specific central circuits, with consequent impact on its therapeutic effectiveness in movement disorders.

Key words: axonal transport; clostridial neurotoxins; dystonia; spasticity; synaptic transmission; transcytosis

\section{Significance Statement}

Botulinum neurotoxins are among the most potent toxins known. Despite this, their specific and reversible action prompted their use in clinical practice to treat several neuromuscular pathologies (dystonia, spasticity, muscle spasms) characterized by hyperexcitability of peripheral nerve terminals or even in nonpathological applications (i.e., cosmetic use). Substantial experimental and clinical evidence indicates that not all botulinum neurotoxin Type A (BoNT/A) effects can be explained solely by the local action (i.e., silencing of the neuromuscular junction). In particular, there are cases in which the clinical benefit exceeds the duration of peripheral neurotransmission blockade. In this study, we demonstrate that BoNT/A is transported to facial motoneurons, released, and internalized preferentially into cholinergic terminals impinging onto the motoneurons. Our data demonstrate a direct central action of BoNT/A.

\section{Introduction}

Botulinum neurotoxin Type A (BoNT/A) is one of the most potent toxins known (Pirazzini et al., 2017). It blocks neurotrans-

Received Jan. 30, 2018; revised Aug. 6, 2018; accepted Aug. 15, 2018.

Author contributions: M.C. and L.R. wrote the first draft of the paper; I.M., O.R., Z.L., and L.R. edited the paper; M.C., I.M., and L.R. designed research; M.C., M.S., F.C., I.M., and L.R. performed research; 0 .R. contributed unpublished reagents/analytic tools; M.C., M.S., F.C., and L.R. analyzed data; M.C., M.S., and L.R. wrote the paper.

This work was supported by Italian Association for Cancer Research Grant IG 18925, CNR (Joint Lab 2018 Project), and Croatian Science Foundation IP-2014-09-4503. We thank Francesca Biondi for excellent animal care; Silvia Pellicciotta for help in protocol illustrations; and Thea Sesardic for providing equine antitoxin to BoNT/A.

The authors declare no competing financial interests.

*M.C. and M.S. contributed equally to this work.

Correspondence should be addressed to either Dr. Matteo Caleo or Dr. Laura Restani, CNR Neuroscience Institute, Pisa 56124, Italy, E-mail: caleo@in.cnr.it or restani@in.cnr.it. mission via the specific cleavage of the synaptic protein SNAP-25 (synaptosomal-associated protein of $25 \mathrm{kDa}$ ). Intramuscular injection of BoNT/A has been a major advance in the therapy of focal dystonia and spasticity, due to the reversible inhibition of hyperexcitable nerve terminals (Simpson et al., 2008). Several reports indicate that clinical benefit does not always parallel the extent of muscle weakness (e.g., the functional improvement may outstrip peripheral neuroparalysis, pointing to central effects of the neurotoxin) (Trompetto et al., 2006; Mazzocchio and Caleo, 2015). One possibility is BoNT/A-mediated blockade of intra- 
fusal fibers, with reduced spindle afferent input to the motoneurons (MNs) (Trompetto et al., 2006). Because BoNT/A is retrogradely transported (Wiegand and Wellhöner, 1977; Antonucci et al., 2008; Restani et al., 2012a), it may also affect central circuits directly via axonal trafficking and transfer to upstream neurons (transcytosis). Indeed, several groups have reported appearance of BoNT/Acleaved SNAP-25 in distant areas following local delivery of the neurotoxin (Restani et al., 2012a; Wang et al., 2015; Bomba-Warczak et al., 2016). However, it remains unclear whether the catalytically active toxin is transported and transcytosed from MNs to functionally connected synapses in vivo. There is also a lack of information on the central neurons that are affected following intramuscular BoNT/A. Here we have exploited an animal model to gain novel insights into the mechanisms and potential therapeutic relevance of BoNT/A central activity.

\section{Materials and Methods}

Ethics statement. All procedures were performed in compliance with the European Union Council Directive 2010/63/EU on the protection of animals used for scientific purposes, and approved by the Italian Ministry of Health (for experiments performed on C57BL/6 mice and Long-Evans rats, Protocol 346/2013-B) and Croatian Ministry of Agriculture (for experiments with Wistar rats, Protocol EP 24-2/2015).

Experimental design and statistical analysis. We injected BoNT/A or onabotulinumtoxin A into the nasolabial musculature (whisker pad [WP]) of rodents to investigate trafficking and effects of the toxins in distant regions, such as the facial nucleus (FN).

All statistical tests were performed using SigmaPlot 12.0 (Systat Software). For comparison between three groups, one-way ANOVA on ranks was used, followed by a Bonferroni's test. Mann-Whitney Rank Sum test was used for comparison between two groups. All the sections collected from animals in each experimental group were pooled together for the statistical analyses ( $\alpha$ value 0.05 ). No samples were excluded from analysis.

Procedures. A total of 45 C57BL6J mice and 15 rats of both sexes (age range: 3-6 months) were used. An initial set of experiments were performed in rats (Long-Evans and Wistar rats); then we turned to mice as this is the species that is currently mostly used to monitor peripheral neuroparalysis and toxicity of BoNTs (Keller, 2006; Morbiato et al., 2007; Kutschenko et al., 2017). We estimated the number of animals to be used for the different experiments based on the results of our previous papers on BoNT/A trafficking in the visual system (Restani et al., 2011, 2012b). All experimental animals were included in the analysis. The number of animals and the species/strain

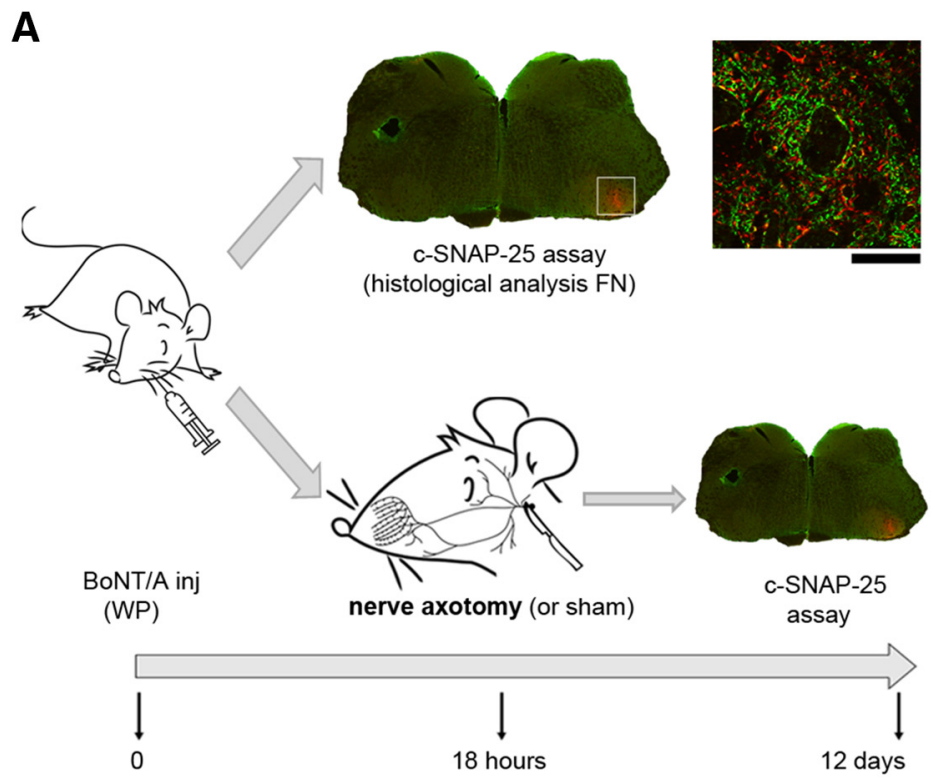

B
18 hours

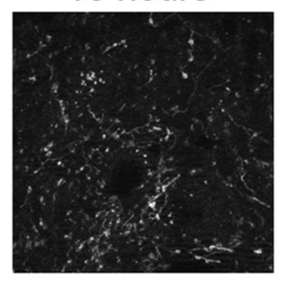

axotomy

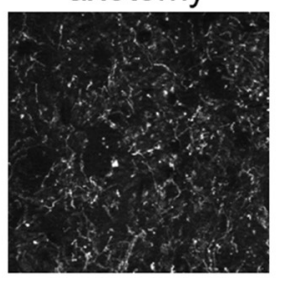

sham

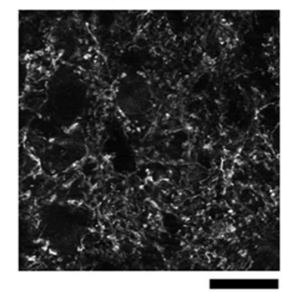

C

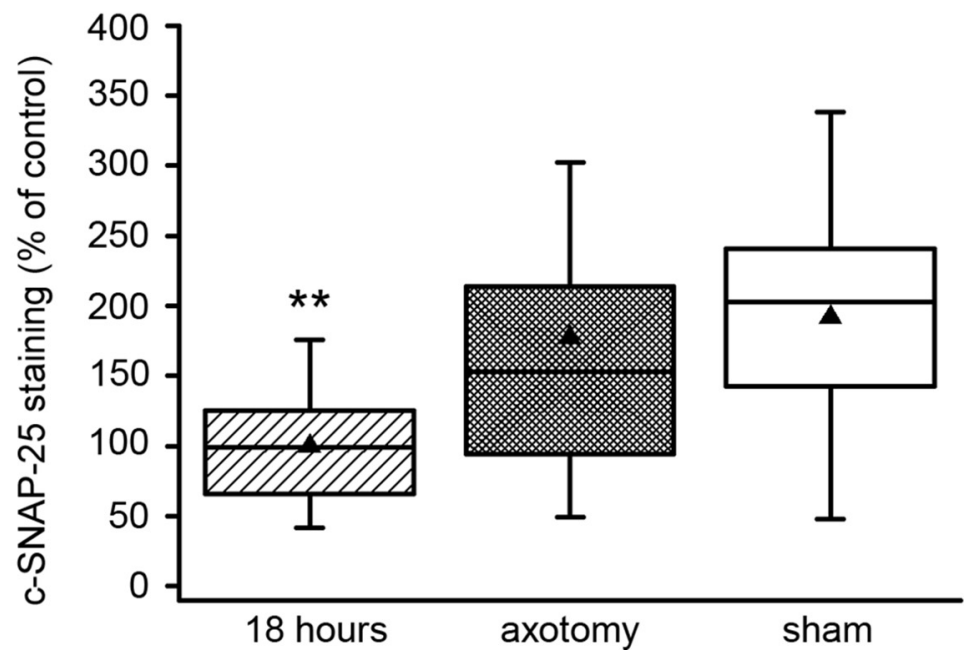

Figure 1. Enzymatically active BoNT/A is retrogradely transported. $\boldsymbol{A}$, Sketch of the experimental protocol: BoNT/A (7.5 pg) was injected into the WP muscles of mice. At $18 \mathrm{~h}, 4$ animals were processed for c-SNAP-25 assessment, whereas the others underwent either facial nerve axotomy (axotomy, $n=5$ ) or sham surgery (sham, $n=5$ ). The site of axotomy is indicated by the blade. Their brains were dissected at day 12. Inset, Double labeling for c-SNAP-25 (red) and the synaptic marker synaptophysin (green). The small puncture on the brain section indicates the side opposite to toxin injection. Scale bar, 25 $\mu \mathrm{m} . \boldsymbol{B}$, Representative images showing the characteristic staining for c-SNAP-25 into the FN for each experimental group. Scale bar, $25 \mu \mathrm{m}$. C, Quantification of c-SNAP-25 immunoreactivity on FN coronal sections (18-21 sections per group). Statistical analysis reveals that levels of c-SNAP-25 increased in the FN despite axotomy (one-way ANOVA on ranks, $p<$ 0.001; post hoc Bonferroni's test, axotomy vs $18 \mathrm{~h},{ }^{* *} p=0.01$ ), indicating that catalytically active BoNT/A is retrogradely transported in MNs in vivo. Intensity of the staining for each experiment was normalized to the labeling levels at $18 \mathrm{~h}$ (control). Horizontal lines in each box indicate the 25th, 50th, and 75 th percentile values. Error bars indicate the fifth and 95 th percentile values. Triangles represent the mean of the column of data. 
rat, BoNT/A 1 day
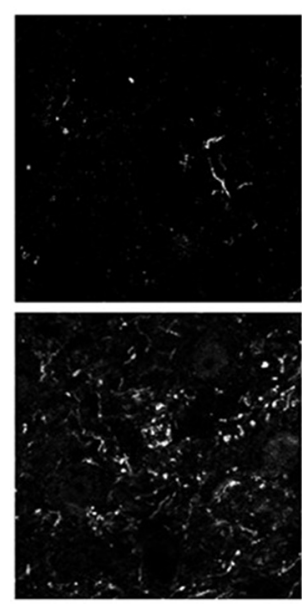

mouse, BoNT/A 1 day rat, BoNT/A 3 days
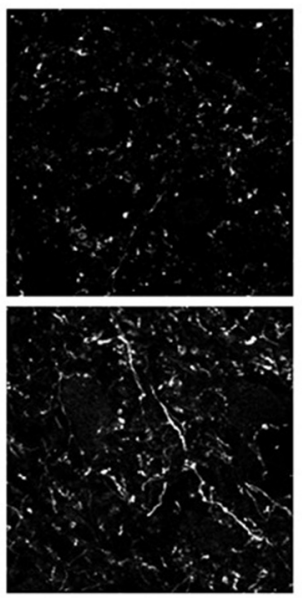

mouse, BoNT/A 3 days rat, BoNT/A 15 days
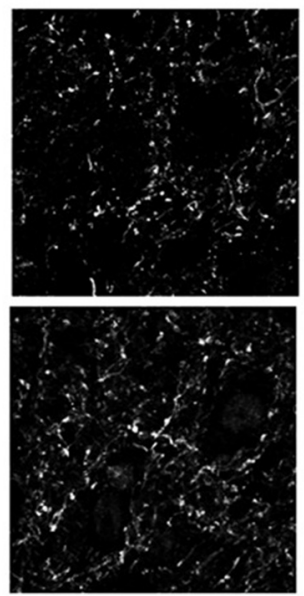

mouse, BoNT/A 15 days
Figure 2. Different kinetics of C-SNAP-25 accumulation in the FN of rats and mice. Top, Representative images showing staining for c-SNAP-25 in the FN of Long-Evans rats at different times following BoNT/A injection ( $75 \mathrm{pg}$ ) into the WP ( $n=3$ rats per time point). Bottom, Representative images of c-SNAP-25 staining in the FN of mice at different times following BoNT/A injection (7.5 $\mathrm{pg}$ ) into the WP ( $n=3$ mice per time point). c-SNAP- 25 is already present at $1 \mathrm{~d}$ in mice but barely detectable in rats. Scale bar, $25 \mu \mathrm{m}$.

A

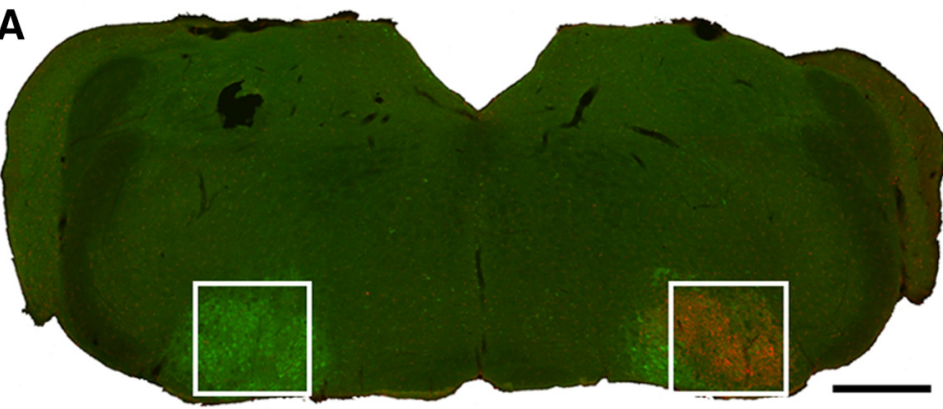

B
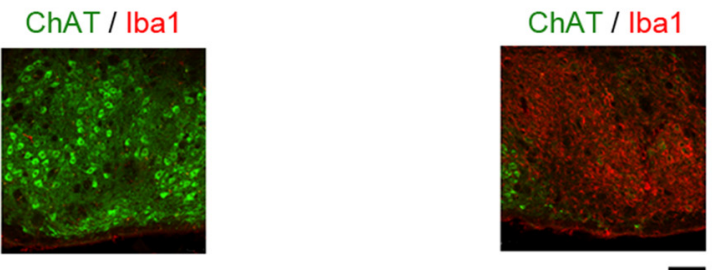

C

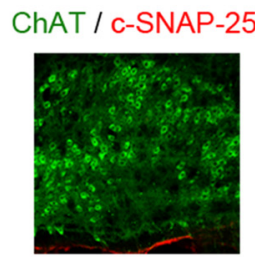

ChAT / c-SNAP-25

Figure 3. Controls for successful axotomy of facial MNs projecting to the WP. Low-magnification image $(\boldsymbol{A})$ and zoom of the two facial nuclei $(\boldsymbol{B})$ of a brainstem coronal section stained for Iba-1 (red) and ChAT (green). In the ipsilesional side (right), note the strong lba-1 immunoreactivity and downregulation of ChAT expression. Despite the axotomy, c-SNAP-25 is strongly expressed in the ipsilesional FN (red staining in $\boldsymbol{C}$ ). Scale bars: $\boldsymbol{A}, 500 \mu \mathrm{m} ; \boldsymbol{B}, \boldsymbol{C}, 100 \mu \mathrm{m}$. Experiments were performed in 4 mice with FN axotomy. used for each experiment are indicated in the figure legends.

BoNT/A injection into the WP, immunohistochemistry for cleaved SNAP-25, and synaptic markers were performed as previously reported (Antonucci et al., 2008; Matak et al., 2011; Restani et al., 2011, 2012a). A laboratory preparation of BoNT/A, lacking accessory proteins (kind gift of C. Montecucco, University of Padua), was injected in Long-Evans rats and mice ( 75 and $7.5 \mathrm{pg}$ in a volume of 500 and 100 $\mathrm{nl}$, respectively). This toxin batch displays an $\mathrm{LC}_{50}$ of $0.25 \mathrm{ng} / \mathrm{kg}$ (Pirazzini et al., 2017). Thus, the amount injected in mice ( $7.5 \mathrm{pg}$ ) corresponds to a single $\mathrm{LC}_{50}$. Signs of botulism were not observed in the injected animals as the delivery is local and restricted in a tiny volume (as previously reported for digital abduction score assay) (Pirazzini et al., 2014). We also used a commercial BoNT/A preparation (onabotulinumtoxin A; Botox, Allergan; $10 \mathrm{U} / \mathrm{kg}$, comparable with the doses recently used in the TOWER study) (Wissel et al., 2017). OnabotulinumtoxinA was delivered in a volume of $20 \mu \mathrm{l}$ in Wistar rats and $1 \mu \mathrm{l}$ in mice. The amount of Botox $(10 \mathrm{U} / \mathrm{kg})$ used in mice was between threefold and fourfold lower than the dose used for laboratory preparation of BoNT/A (considering the weight of our adult C57BL6 J mice $=25-30 \mathrm{~g}$ ).

We exploited an equine neutralizing monovalent antitoxin (Botulinum Type A antitoxin from National Institute for Biological Standards and Control, NIBSC code 14/174, Potters Bar, United Kingdom; a kind gift from Dr. Thea Sesardic) raised against the whole BoNT/A molecule, thus binding both light and heavy chain. It was delivered by intracerebroventricular injections in mice ( $2 \mathrm{IU}$ in $2 \mu \mathrm{l}$ sterile saline) or by percutaneous injection into the cisterna magna in rats (20 IU in $20 \mu \mathrm{l}$ saline); 1 IU of equine monovalent antitoxin is able to neutralize 10,000 mouse LD 50 units of BoNT/A. The doses of antitoxin were chosen based on the low penetrability of IgGs into the CNS tissue. Facial nerve axotomy was performed as previously described (Serpe et al., 1999).

Antibodies used for double labeling of synaptic terminals were as follows: goat polyclonal anti-VAChT (1:1000, Millipore), mouse monoclonal anti-VGlut-2 (1:250, Millipore), and guinea pig anti-VGAT (1:300, Synaptic Systems). The antibody against cleaved SNAP- 25 is a custom-made antibody produced in the laboratory of Dr. Ornella Rossetto in Padua, and characterized by several previous studies (Antonucci et al., 2008; Matak et al., 2011; Restani et al., 2011, 2012a,b).

Sections were scanned with a microscope (Axio Imager.Z2, Carl Zeiss) equipped with Apotome.2 (Carl Zeiss) at resolutions of $1024 \times 1024$ pixels $(63 \times$ EC-Plan-Neofluar oil objective, NA 1.4). Quantification of the number of terminals positive for cleaved SNAP-25or other presynaptic markers was performed as previously described (Ippolito and Eroglu, 2010), using Fiji software (Schindelin et al., 2012) and Puncta analyzer plugin (Ippolito and Eroglu, 2010; Baroncelli et al., 2016). 
A

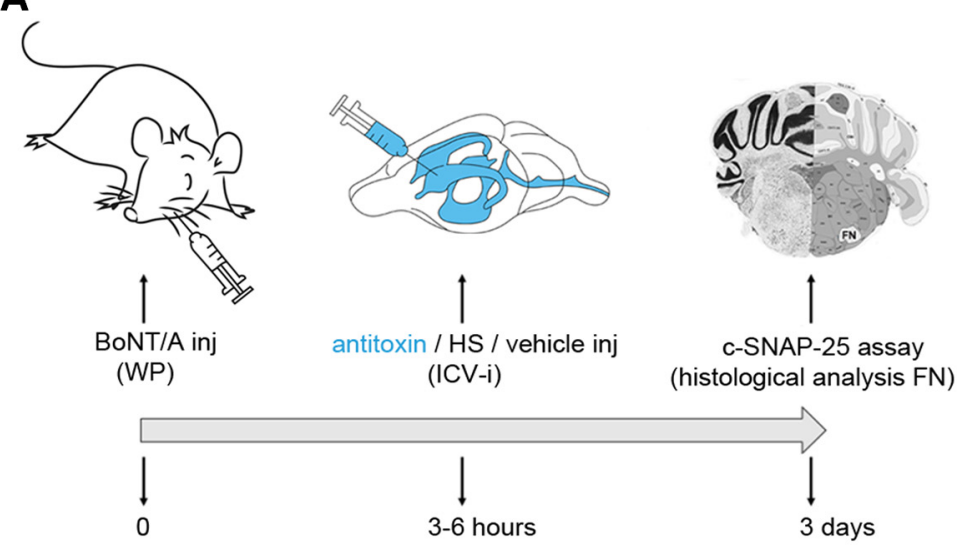

B
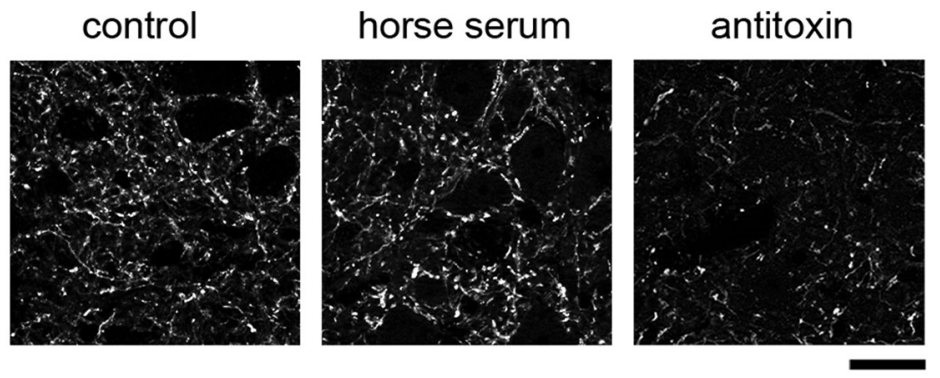

C

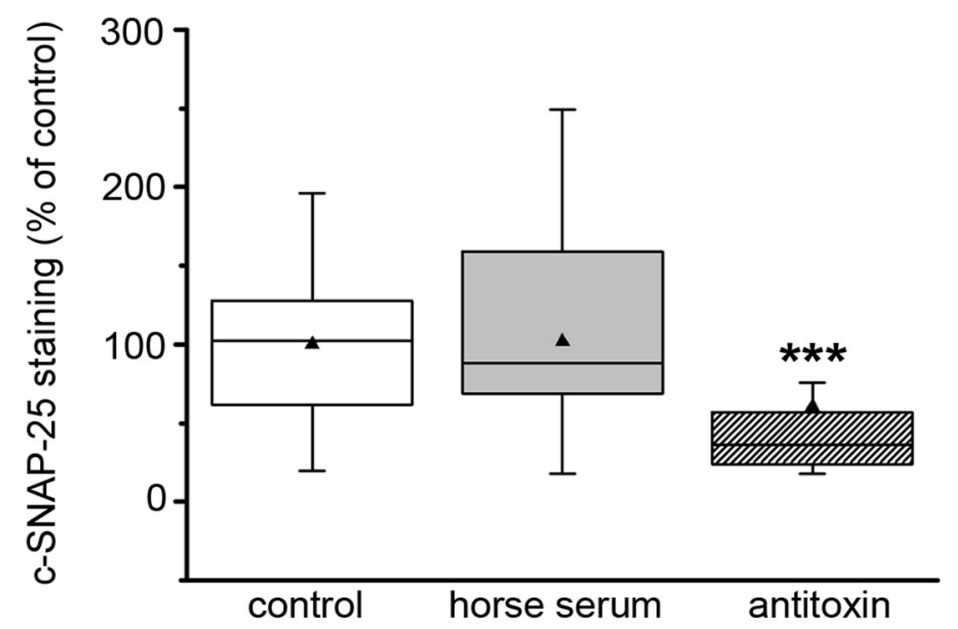

D
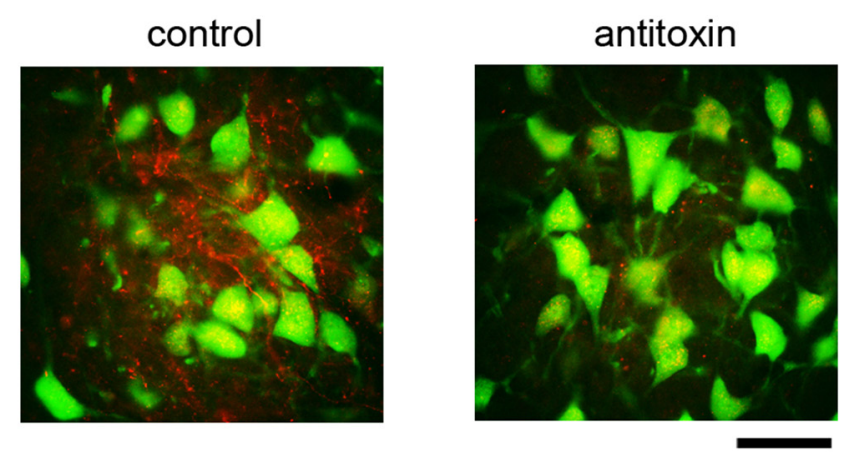

Figure 4. Evidence for BoNT/A transcytosis. $\boldsymbol{A}$, Sketch of the experimental protocol: BoNT/A (7.5 pg) was injected into the WP muscles of mice. After 3-6h, mice received antitoxin, horse serum (HS), or vehicle solution (control) into the ventricular system. Mice were killed after $3 \mathrm{~d}$ and brains dissected for histological analysis. ICV-i, Intracerebroventricular injection. $\boldsymbol{B}$, Representative

\section{Results}

Evidence for transport of catalytically active BoNT/A in MNs

We used immunostaining to define the time course of SNAP-25 cleavage in the FN of rats and mice following intramuscular injection into the WP (Antonucci et al., 2008; Restani et al., 2012a). Labeling for cleaved SNAP-25 (c-SNAP-25) was specifically detected only in the FN ipsilateral to the injection (Fig. 1A). In rats, immunoreactivity was almost undetectable at day 1 and substantially increased between days 3 and 15 (Fig. 2, top). In mice, the accumulation of c-SNAP-25 was faster (possibly related to the shorter length of facial axons); indeed, it was already visible at $1 \mathrm{~d}$ and increased until $15 \mathrm{~d}$ (Fig. 2, bottom).

To determine whether the accumulation of c-SNAP-25 was due to local proteolysis within the $\mathrm{FN}$ (rather than to retrograde transport of the cleaved substrate from the WP), we examined the buildup of c-SNAP-25 after interruption of axonal transport by facial nerve axotomy. BoNT/A was injected into the WP of a group of mice. At $18 \mathrm{~h}, 4$ animals were killed, whereas the remaining underwent either facial nerve axotomy (axotomy) or sham surgery (sham) and their brains were dissected at day 12 (Fig. 1A).

Representative labeling for c-SNAP-25 in the $\mathrm{FN}$ of the three experimental groups is shown in Figure 1B. The staining

\section{$\leftarrow$}

images showing staining for C-SNAP-25 in the FN of control, HS-treated, and antitoxin-treated animals. Note the clear reduction in C-SNAP-25 immunoreactivity when antitoxin is delivered into cerebral fluid. Scale bar, $25 \mu \mathrm{m}$. C, Quantification of c-SNAP-25 immunoreactivity on FN coronal sections (control, $n=13$ mice; HS, $n=5$; antitoxin $n=11$ ). Statistical analysis reveals that levels of c-SNAP-25 decreased by almost half following intracerebroventricular injection of antitoxin, but not HS (one-way ANOVA on ranks followed by Bonferroni's test, $\left.{ }^{* * *} p<0.001\right)$. Data indicate that at least a fraction of c-SNAP-25 in the FN depends on BoNT/A transcytosis. Horizontal lines in each box indicate the 25 th, 50 th, and 75 th percentile values. Error bars indicate the fifth and 95th percentile values. Triangles represent the mean of the column of data. $\boldsymbol{D}$, Representative images showing staining for C-SNAP-25 in the facial nuclei following two separate injections into the WP (10 $\mathrm{U} / \mathrm{kg}$ ) of Wistar rats $(n=5)$. OnabotulinumtoxinA was administered into the WP $5 \mathrm{~d}$ (right WP) and $1 \mathrm{~d}$ (left WP) before intracisternal injection of botulinum Type $A$ antitoxin (20 IU). c-SNAP-25 staining (red) is basically absent in the FN ipsilateral (left FN) to BoNT/A injection administered $1 \mathrm{~d}$ before antitoxin (i.e., BoNT/A + antitoxin $1 \mathrm{~d}$ later), although it is normally present ipsilateral to BoNT/A administered $5 \mathrm{~d}$ before antitoxin (right FN, i.e., BoNT/A + antitoxin 5 d later). The FN images are from the two sides of the same brainstem section. Green staining represents neuronal marker (NeuN). Scale bar, $100 \mu \mathrm{m}$. 
A
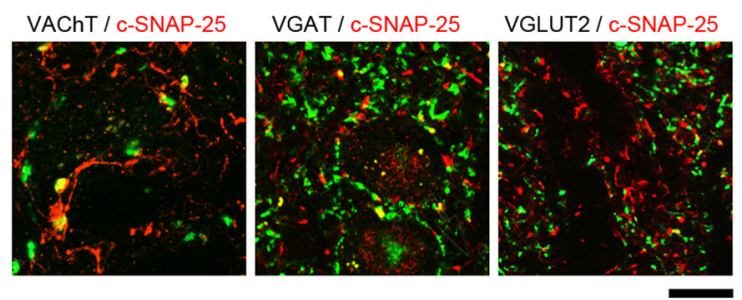

B

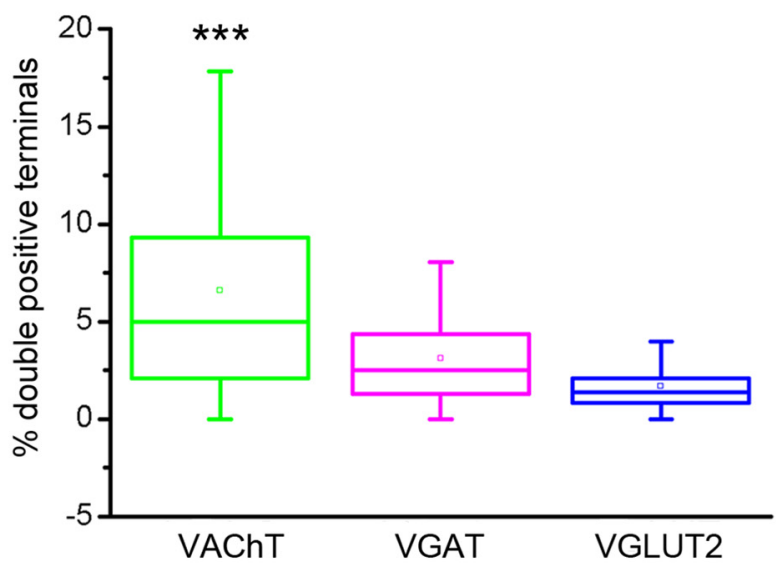

C

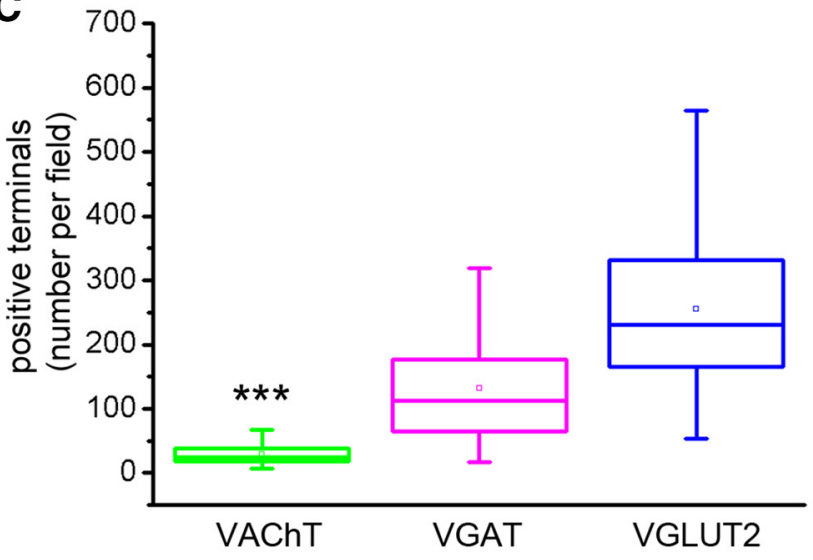

D
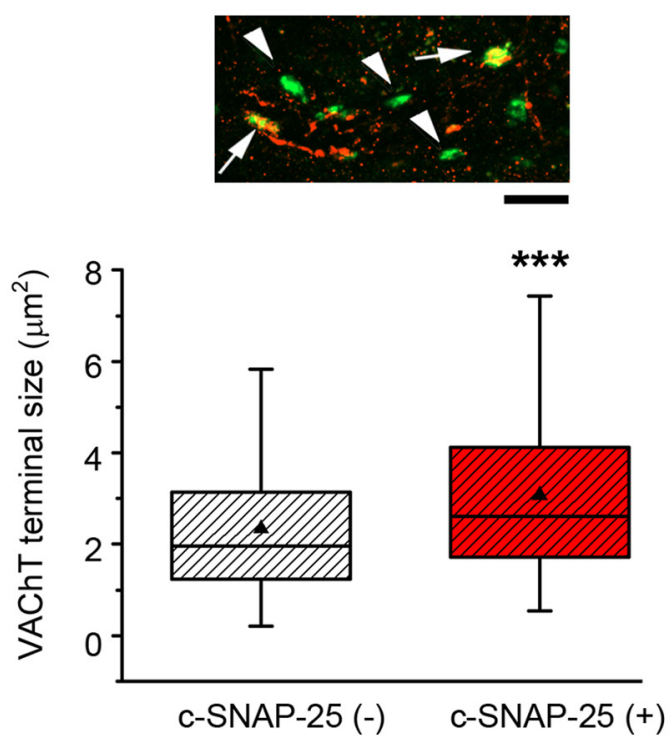

Figure 5. Central cholinergic terminals are preferentially targeted. $\boldsymbol{A}$, Representative images showing double-staining for c-SNAP-25 (red) and specific synaptic markers (VAChT, VGAT, and VGLUT-2; green) in the FN. BoNT/A injection into the WP of Long-Evans rats $15 \mathrm{~d}$ earlier. Scale bar, $15 \mu \mathrm{m}$. $\boldsymbol{B}$, Colocalization analysis in FN coronal sections $15 \mathrm{~d}$ following BoNT/A appeared mainly in fiber-like structures, processes, and synaptic terminals (as shown by colocalization with the synaptic marker synaptophysin; Fig. 1A, inset). Quantitative analysis indicates that levels of c-SNAP-25 dramatically increased in the FN despite the axotomy (one-way ANOVA on ranks, $F_{(2,59)}=8.86$; followed by Bonferroni's test: $18 \mathrm{~h}$ vs axotomy, $p=0.010$; sham vs $18 \mathrm{~h}$, $p<0.001$; Fig. $1 C$ ) and were indistinguishable from the sham group at day $12(p=1.000)$. The buildup of c-SNAP-25 in mice with facial nerve axotomy can only be explained by the retrograde trafficking of the catalytically active protease during the first $18 \mathrm{~h}$ after the injection.

To confirm that the facial nerve axotomy was complete, we evaluated markers of MNs and gliosis in the same animals used for c-SNAP-25 quantification. Specifically, we examined expression of ChAT (the biosynthetic enzyme for acetylcholine) and ionized calcium-binding adapter molecule 1 (Iba-1), a protein expressed in microglia. As expected, in the ipsilesional (right) side, we detected a profound downregulation of ChAT in MNs projecting to the WP and a corresponding upregulation of Iba-1, indicative of reactive gliosis (Fig. $3 A, B$ ) (Hottinger et al., 2000; Moran and Graeber, 2004). Within the medial part of the FN, only a small subset of MNs projecting to the auricular musculature (Ashwell, 1982) were found to retain ChAT expression, whereas the lateral MNs showed almost complete loss of ChAT, indicative of successful axotomy (Fig. 3A,B). High levels of c-SNAP-25 were found specifically in the lateral aspect of the FN, which contains the axotomized MNs projecting to the nasolabial musculature (Ashwell, 1982) and displaying strong ChAT downregulation (Fig. 3C).

\section{Evidence for BoNT/A transcytosis}

After retrograde transport, catalytically active BoNT/A may remain confined within the MNs or undergo transcytosis into upstream neurons, similar to tetanus neurotoxin. We reasoned that, if BoNT/A is released from MNs after retrograde trafficking, it should be neutralized by antitoxin antibodies circulating in the extracellular space. Accordingly, we injected BoNT/A into the WP of a group of mice, and 3-6 h later we delivered into the cerebral fluid either an antiserum specific for BoNT/A (antitoxin) or vehicle, via intracerebroventricular injection. Three days after intracerebroventricular injection, antitoxin $(n=11)$ and vehicle animals $(n=13)$ were perfused and analyzed for c-SNAP-25. As a further control, we injected normal horse serum instead of antitoxin $(n=5)$ (Fig. $4 A)$. Quantification revealed that intracerebroventricular injection of the antitoxin is very potent in reducing the levels of c-SNAP-25 in the FN, whereas ad-

\footnotetext{
$\leftarrow$

injection into rat WP (98-131 sections per synaptic marker, 4 rats). Statistical analysis reveals that the percentage of VAChT-positive boutons containing C-SNAP-25 was significantly higher than the percentage calculated for VGAT and VGLUT2 (one-way ANOVA on ranks; post hoc Bonferroni's test, VAChT vs VGAT e VGLUT2, ${ }^{* * *} p<0.001$ ). C, Data represent the total number of positive puncta per field for each synaptic marker analyzed. Statistical analysis reveals highest staining for VGLUT2, followed by VGAT and VAChT (one-way ANOVA on ranks; post hoc Bonferroni's test, VAChT vs VGAT e VGLUT2, ${ }^{* * *} p<0.001$ ). Each field used for the analysis was $104 \times 104 \mu \mathrm{m}$. D, Quantification of VAChT terminal size, measured in boutons negative $(-)$ or positive ( + ) for c-SNAP-25 (472 and 354 boutons analyzed, respectively; $n=3$ Long-Evans rats). VAChT terminals intoxicated by BoNT/A (c-SNAP-25 (+)) display a larger size with respect to controls (Mann-Whitney Rank Sum test, ${ }^{* * *} p<0.001$ ). Inset, Representative high magnification of double labeling for VAChT (green) and c-SNAP-25 (red). Arrows indicate doublepositive boutons. Arrowheads indicate c-SNAP-25(-) terminals. $\boldsymbol{B}-\boldsymbol{D}$, Horizontal lines in the box charts indicate the 25 th, 50 th, and 75 th percentile values. Error bars indicate the fifth and 95th percentile values. Triangles represent the mean of the column of data.
} 
ministration of horse serum had no effect, ruling out unspecific effects due to antibody delivery (one-way ANOVA on ranks, $F_{(2,85)}=11.93$; followed by Bonferroni's test: control vs antitoxin, $p<0.001$; control vs horse serum, $p=1.000$; horse serum vs antitoxin, $p=0.001$; Fig. $4 B, C)$. These results demonstrate release of active BoNT/A from the transporter MNs within the FN.

We performed similar experiments with a therapeutic dose $(10 \mathrm{U} / \mathrm{kg})$ (Wissel et al., 2017) of a commercial preparation of BoNT/A (onabotulinumtoxinA, 900 $\mathrm{kDa}$ complex) in rats $(n=5)$. We injected the toxin unilaterally into the right WP of animals; and after allowing $5 \mathrm{~d}$ for transport and transcytosis to occur in the right $\mathrm{FN}$, we inoculated onabotulinumtoxinA into the left WP of the same animals. This second injection was followed next day by delivery of antitoxin into the CSF via the cisterna magna, and the animals were killed at day 15 . We found that, while the right $\mathrm{FN}$ displayed typical staining for c-SNAP-25, the cleaved substrate was basically undetectable in the left FN, consistent with the idea that the antitoxin neutralizes the fraction of toxin that is released from transporter MNs into the extracellular fluid before transcytosis entry into secondary synapses (Fig. $4 D$ ).

Preferential SNAP-25 cleavage in cholinergic terminals

We next analyzed the types of central nerve terminals containing c-SNAP-25 (i.e., targeted by BoNT/A) by performing doublelabel immunohistochemistry for c-SNAP-25 and specific synaptic markers (vesicular transporters for glutamate Type 2, GABA/ glycine, and acetylcholine vesicular glutamate transporter 2 [VGLUT2], VGAT, VAChT), $15 \mathrm{~d}$ after BoNT/A injection into rat WP (Fig. 5A).

We segmented the labeled neuropil pixels into presumptive synaptic sites (Ippolito and Eroglu, 2010) (see Materials and Methods) and quantified the numbers of single- and doublelabeled terminals. We found that the percentage of boutons double-positive for c-SNAP-25 and VAChT was significantly higher than for c-SNAP-25/VGAT or c-SNAP-25/VGLUT2 (one-way ANOVA on ranks, $F_{(2,344)}=33.58$; followed by Bonferroni's test, $p<0.001$; Fig. $5 B$ ). This was striking as cholinergic profiles were relatively rare compared with GABAergic and glutamatergic terminals (one-way ANOVA on ranks, $F_{(2,342)}=$ 484.92; followed by Bonferroni's test, $p<0.001$; Fig. $5 C)$. Thus, BoNT/A appears to preferentially target cholinergic terminals following retrograde transport from the rat WP.

We also examined morphological abnormalities in cholinergic terminals positive for c-SNAP-25. Previous studies have demonstrated an enlargement of central synapses exposed to BoNT/A, possibly as a result of vesicle accumulation (Caleo et al., 2012; Restani et al., 2012b). We compared synaptic terminal size of VAChT boutons positive or negative for c-SNAP-25 within the same FN sections (Fig. 5D, inset). We found that terminals doublelabeled for c-SNAP-25 and VAChT were correlated with a larger
B

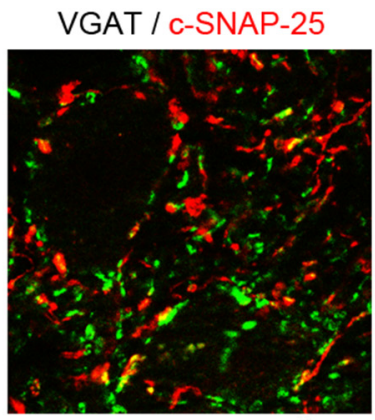

E

GlyT2 / c-SNAP-25

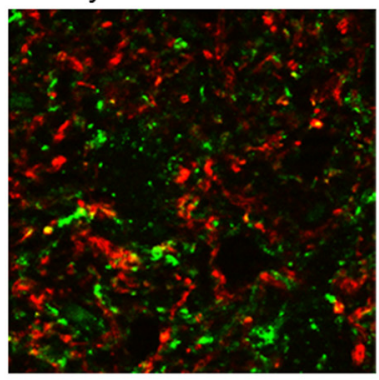

$\mathbf{F}$

VGLUT1 / c-SNAP-25

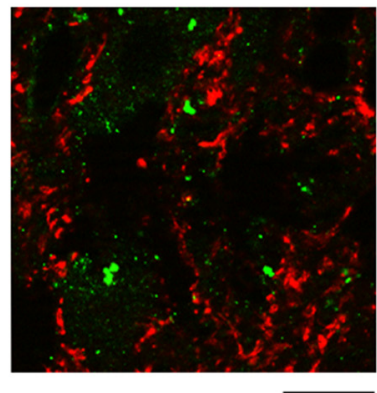

VGLUT2 / c-SNAP-25

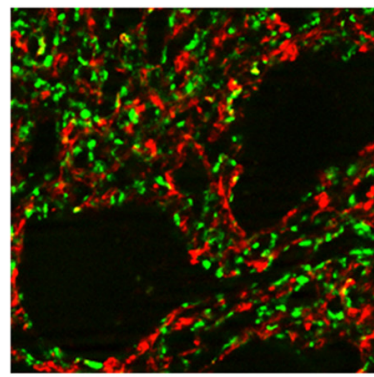

(2010

Figure 6. Neurochemical specificity of cleaved SNAP-25-positive structures in the mouse FN. BoNT/A was injected into the use WP, and brains were taken at $12 \mathrm{~d}$. Immunostaining in FN sections for cleaved SNAP-25 (red) and synaptic markers (green) ,E, GlyT2.F, VGLUT1. Scale bar, $15 \mu \mathrm{m}$.

terminal size compared with cholinergic boutons not containing c-SNAP-25 (Mann-Whitney Rank Sum test, $p<0.001$; Fig. 5D).

We went on to perform a more comprehensive analysis of the expression of BoNT/A-truncated SNAP-25 in distinct types of synaptic boutons after BoNT/A delivery to the mouse WP (Fig. 6). In addition to VAChT-, vGAT-, and vGLUT2-positive boutons (Fig. $6 A-C$ ), we examined the expression of cleaved SNAP-25 in serotonergic and glycinergic terminals that are highly expressed in the FN. Specifically, we used antibodies against the serotonin transporter (SERT) and glycine transporter 2 (GlyT2) to reveal a potential action of BoNT/A on these terminals. We found basically no costaining of SERT and GlyT2 with BoNT/A-truncated SNAP-25 (Fig. 6D,E). To shed further light on the possible intoxication of excitatory glutamatergic terminals, we stained for VGLUT1. We found very little expression of VGLUT1 (similar to VAChT) in the FN but virtually no colocalization with cleaved SNAP-25 (Fig. 6F).

It was important to check whether a commercial preparation of BoNT/A (onabotulinumtoxinA, $900 \mathrm{kDa}$ complex) displays the same preferential activity at central cholinergic boutons. Thus, we inoculated mice with onabotulinumtoxinA $(10 \mathrm{U} / \mathrm{kg})$ into the WP and examined c-SNAP-25-positive synaptic terminals in the FN at day 12 (Fig. $7 A$ ). The results were very similar to those obtained with the laboratory BoNT/A preparation. Indeed, the VAChT-positive boutons showed the highest percentage of colocalization with c-SNAP-25, far exceeding that measured for VGAT and VGLUT2 (one-way ANOVA on ranks, $F_{(2,91)}=$ 113.16; followed by Bonferroni's test, $p<0.001$; Fig. $7 B$ ). This is remarkable as both VGAT- and VGLUT2-positive terminals clearly outnumbered cholinergic profiles, which were typically very sparse in the neuropil (one-way ANOVA on ranks, $F_{(2,91)}=$ 94.05; followed by Bonferroni's test, $p<0.001$; Fig. 7 A, $C$ ). 
A
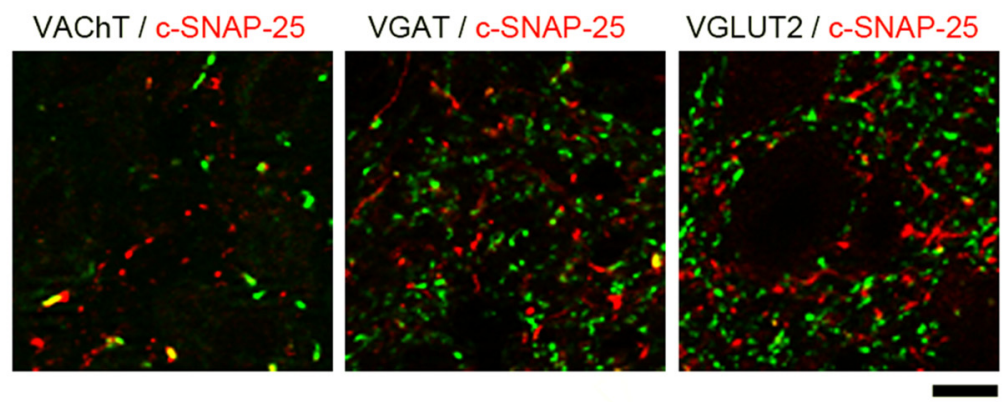

B

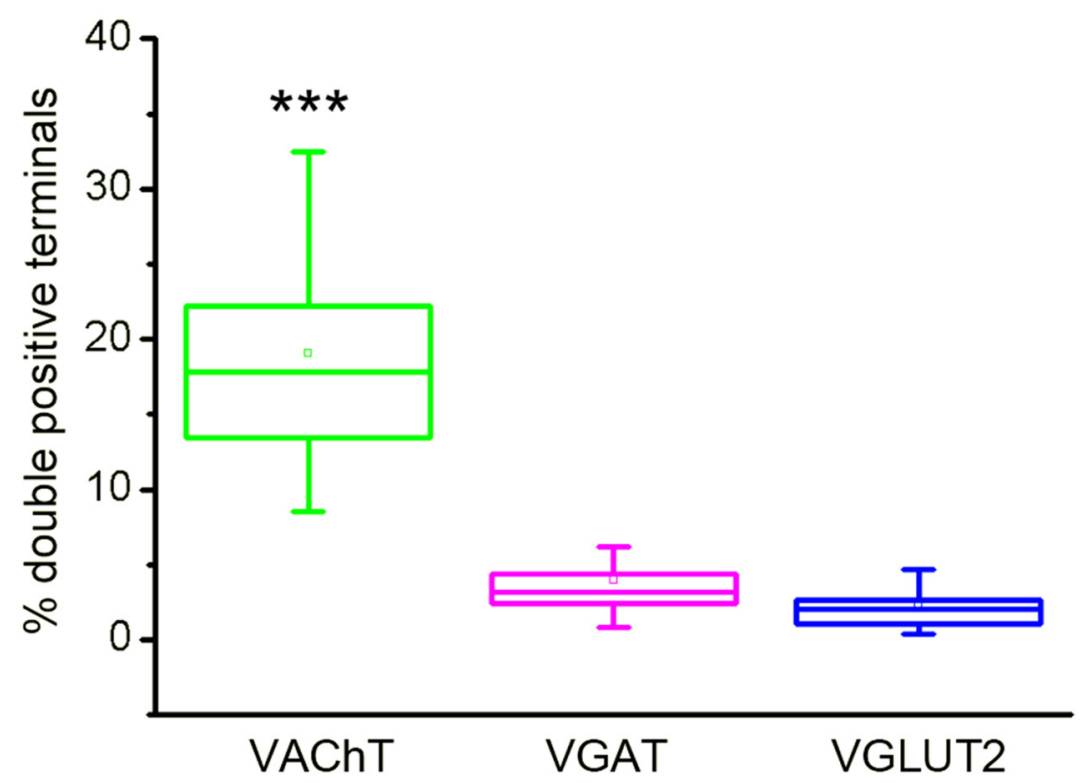

C

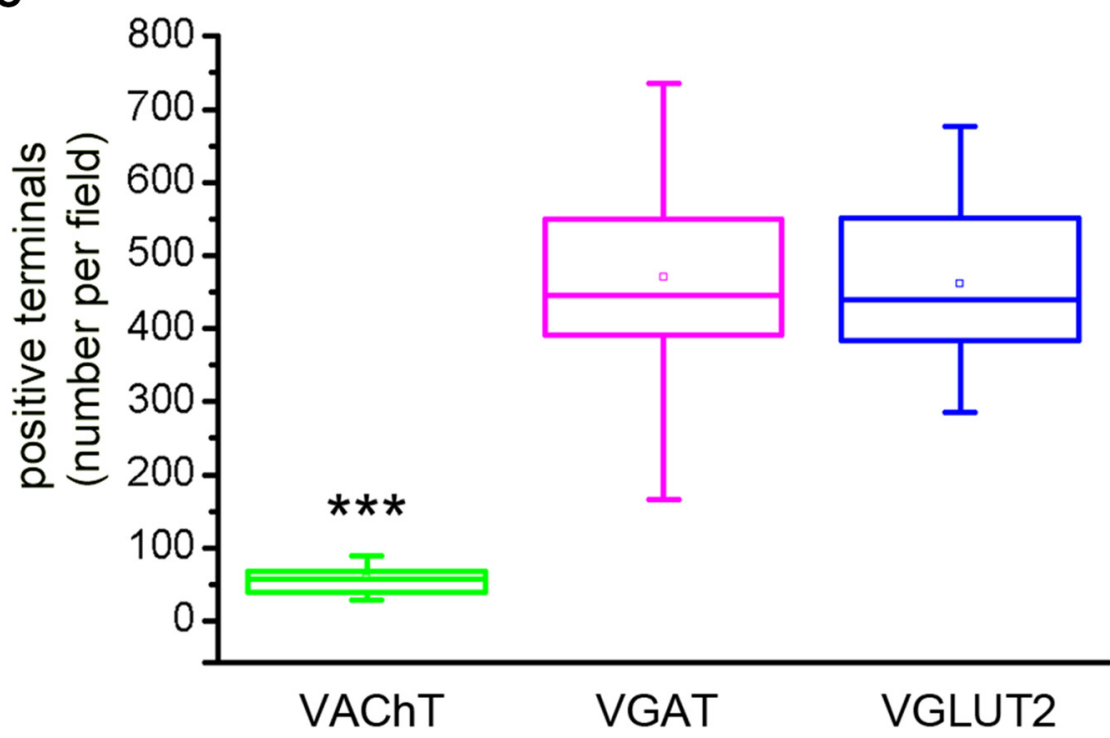

Figure 7. Preferential activity of onabotulinumtoxinA at central cholinergic synapses. $A$, Representative images of the FN showing double-staining for c-SNAP-25 (red) and specific synaptic markers (VAChT, VGAT, and VGLUT-2; green) following onabotulinumtoxinA delivery $(10 \mathrm{U} / \mathrm{kg})$ in the mouse WP. Scale bar, $10 \mu \mathrm{m} . \boldsymbol{B}$, Colocalization analysis in FN coronal sections $12 \mathrm{~d}$ following onabotulinumtoxinA injection into mouse WP (32 sections per synaptic marker, 4 mice). Statistical analysis reveals that the percentage of VAChT-positive boutons containing C-SNAP-25 was significantly higher than the percentage calculated for VGAT and VGLUT2 (one-way ANOVA on ranks; post hoc Bonferroni's test, VAChT vs VGAT and VGLUT2, $\left.{ }^{* * *} p<0.001\right)$. C, Data represent total number of positive puncta per field for each synaptic marker analyzed ( $n=4$ mice). Statistical analysis reveals highest staining for VGLUT2 and VGAT, followed by VAChT (one-way ANOVA on ranks; post hoc Bonferroni's test, VAChT vs VGAT and VGLUT2, ${ }^{* * *} p<0.001$ ). Each field used for the analysis was $104 \times 104$ $\mu \mathrm{m} . \boldsymbol{B}, \boldsymbol{C}$, Horizontal lines in the box charts indicate the 25th, 50th, and 75th percentile values. Error bars indicate the fifth and 95th percentile values. Triangles represent the mean of the column of data.

\section{Discussion}

BoNT/A is widely and successfully used to treat several human pathologies characterized by hyperexcitability of peripheral nerve terminals (Pellett, 2012; Pirazzini et al., 2017). The rationale for BoNT/A use is that, under conditions of increased muscle tone, delivery of the toxin alleviates the pathological symptoms via blockade of neuromuscular transmission. However, substantial experimental and clinical evidence indicates that not all BoNT/A effects can be explained solely by the silencing of the neuromuscular junction (Trompetto et al., 2006; Vinti et al., 2012; Aymard et al., 2013; Marchand-Pauvert et al., 2013). Studies have reported effects at the level of spinal and brain circuits, which likely contribute to the therapeutic benefits (Vinti et al., 2012; Aymard et al., 2013; Marchand-Pauvert et al., 2013; Mazzocchio and Caleo, 2015). There is no consensus, however, on how these central actions arise.

Here we demonstrate in a purely motor system (facial MNs innervating the nasolabial musculature, which lack direct proprioceptive and sensory innervation as well as recurrent collaterals) that BoNT/A: (1) is retrogradely trafficked to brainstem MNs, retaining its catalytic activity; (2) is released within the FN to enter upstream neurons; and (3) preferentially targets central cholinergic synapses. These findings were replicated using both a laboratory and commercial preparation of the toxin (i.e., in the absence or presence of neurotoxin-associated proteins), and either testing a higher and lower (therapeutic) toxin dose, respectively.

We found robust accumulation of c-SNAP-25 into the mouse FN despite axotomy performed $18 \mathrm{~h}$ after intramuscular injection (Fig. 1). This finding can only be explained by transport of BoNT/A during the first hours after injection and maintenance of its catalytic activity within the FN. Furthermore, delivery of antitoxin into the CSF robustly reduced the levels of BoNT/A-truncated SNAP-25 (Fig. 4), indicating that BoNT/A physically leaves MNs and enters second-order neurons to cleave SNAP-25 (Fig. 8). One might argue that the antitoxin could penetrate into neurons, and block the activity of protease intracellularly. However, this scenario is implausible as antitoxin penetration only occurs when the cell membrane is disrupted by pore-forming procedures (Bartels et al., 1994).

To prevent transcytosis, we chose to deliver antibodies against BoNT/A at a time preceding the appearance of SNAP-25 
A

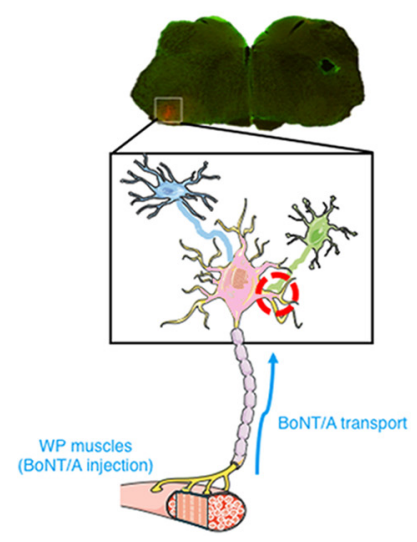

B

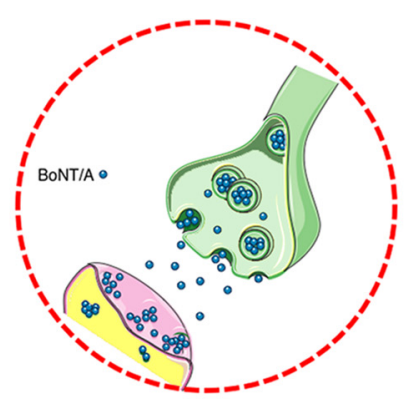

C

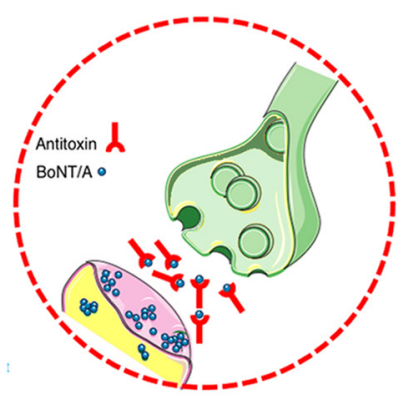

Figure 8. Schematic illustration of the distant effects of BoNT/A involving transcytosis into premotoneuronal synapses. $A$, Sketch of a facial MN projecting to the BoNT/A-injected WP and of its afferent inputs within the FN. $\boldsymbol{B}$, Under control conditions, the retrogradely transported BoNT/A undergoes transcytosis from the MN soma (pink) to the afferent cholinergic synapses (green), leading to a second cycle of intoxication. C, Following intracerebroventricular administration of antitoxin, the released BoNT/A is sequestered within the interstitial space, thus effectively preventing its transfer to afferent boutons.

cleavage (i.e., 3-6 h in mice, $1 \mathrm{~d}$ in rats). We chose to inject a high dose of antitoxin because the delivery occurred via injection into the lateral ventricles, which are far from the target area (the FN in the brainstem). Thus, it is likely that the amount of antitoxin that reaches the interstitial space surrounding facial MNs is relatively low because of both distance and dilution into the cerebral fluids. The fact that antitoxin injection strongly, but not completely, prevented SNAP-25 cleavage (Fig. $4 B, C$ ) may be explained by the administration of a single dose of the antibodies, which may be cleared up from the CSF and the extracellular space, hence not covering the whole time window during which transcytosis occurs. Transcytosis was apparent also at low, therapeutic toxin doses as SNAP-25 cleavage in FN following onabotulinumtoxinA in the WP $(10 \mathrm{U} / \mathrm{kg})$ was blocked by antitoxin delivery. While we cannot exclude that at least part of c-SNAP-25 is present in the somatodendritic compartment of MNs, the antitoxin and facial nerve axotomy experiments clearly show transfer of active BoNT/A to premotoneuronal synapses. Overall, the present data rule out that BoNT/A and its cleaved substrate remain exclusively restricted to the MNs (Cai et al., 2017).

We favor the idea that trans-synaptic transfer happens with the whole BoNT/A molecule. Indeed, data in vitro (Restani et al., 2012a) demonstrate that retrograde trafficking of BoNT/A occurs in vesicles that escape acidification, and consequently this avoids breaking of disulfide bond and release of the light chain. Moreover, it would be impossible for the sole light chain to enter into the presynaptic terminal of second-order neurons because the heavy chain contains the domain responsible for binding to the presynaptic terminal (Herreros et al., 1997; Pirazzini et al., 2017). Overall, the available data strongly suggest that the retrogradely transported BoNT/A molecules escaping acidification are transcytosed as full-length toxins, to allow a second cycle of presynaptic terminal intoxication.

Cleavage of SNAP-25 appeared preferentially in cholinergic terminals despite their relative low density in the FN. The molecular mechanisms underlying this cholinergic selectivity are currently not known but could be due to the expression of specific high-affinity receptors on the plasma membrane of these neurons. Of note, preferential targeting of cholinergic synapses was previously found after long-distance transport of BoNT/A to the retina (Antonucci et al., 2008; Restani et al., 2012b). Importantly, cholinergic terminals containing c-SNAP-25 display a larger size (Fig. 5D). We speculate that the enlargement of cleaved SNAP- 25-positive cholinergic boutons might be due to a continuing supply of mature vesicles arriving at synapses and unable to fuse with the plasma membrane to release their neurotransmitter. Previous studies have shown that the increased terminal size of BoNT/A-intoxicated synapses correlates with enlarged boutons and vesicle accumulation seen with electron microscopy (Caleo et al., 2012; Restani et al., 2012b). Moreover, we previously demonstrated functional synaptic blockade of cholinergic terminals in an ex vivo retinal preparation following retrograde BoNT/A trafficking in the visual pathway (Restani et al., 2012b).

Cholinergic terminals impinging on MNs are known as C-boutons and mediate excitatory input to the MNs (Miles et al., 2007; Witts et al., 2014). They have been identified in the trigeminal, facial, and hypoglossal motor nuclei but not onto other classes of brainstem MNs (e.g., those innervating ocular muscles) (Witts et al., 2014). One possibility is that inhibitory synapses represent an alternative potential target of BoNT/A central action in these motor nuclei. This interpretation is in keeping with recordings of the activity of abducens MNs following BoNT/A delivery into the lateral rectus muscle in cats (Moreno-López et al., 1994). The authors found abnormally low MN discharges together with ultrastructural modifications of afferent synapses (Moreno-López et al., 1997; Pastor et al., 1997). It is worth noting that tetanus neurotoxin, which shares the same axonal carriers as BoNT/A (Restani et al., 2012a; Wang et al., 2015), is also transcytosed into inhibitory interneurons.

The preferential targeting of central cholinergic terminals suggests two sources of reduced $\mathrm{MN}$ output after intramuscular BoNT/A: (1) peripheral neuroparalysis and (2) reduced MN excitability via central effect on cholinergic afferents. In particular, the decreased MN excitability through transcytosis and block of presynaptic excitatory terminals may allow clinical benefits, even when the neuromuscular blockade is over (Valls-Sole et al., 1991; Trompetto et al., 2006; Mazzocchio and Caleo, 2015). If the same scenario applies to spinal cord, a reduction in the $\mathrm{MN}$ firing rate could be envisaged, with consequent decrease in Renshaw cell inhibition via the $\mathrm{MN}$ recurrent collaterals. Reduced recurrent inhibition after peripheral BoNT/A has indeed been demonstrated in humans and animals (Wiegand and Wellhöner, 1977; Marchand-Pauvert et al., 2013). A reduction in the activity of the Renshaw cell network may be crucial for normalizing dysfunctions in the pattern of agonist/antagonist activity in spasticity and 
dystonia (Aymard et al., 2013; Marchand-Pauvert et al., 2013; Mazzocchio and Caleo, 2015).

Together, these findings highlight cell-specific, direct central actions of BoNT/A, which are important to fully understand its mechanisms of action and therapeutic effectiveness in movement disorders. Detailed knowledge of BoNT/A effects on brainstem and spinal pathways is mandatory to devise a mechanism-based use of the neurotoxin, which translates into more effective therapies for patients.

\section{References}

Antonucci F, Rossi C, Gianfranceschi L, Rossetto O, Caleo M (2008) Longdistance retrograde effects of botulinum neurotoxin A. J Neurosci 28: 3689-3696. CrossRef Medline

Ashwell KW (1982) The adult mouse facial nerve nucleus: morphology and musculotopic organization. J Anat 135:531-538. Medline

Aymard C, Giboin LS, Lackmy-Vallée A, Marchand-Pauvert V (2013) Spinal plasticity in stroke patients after botulinum neurotoxin A injection in ankle plantar flexors. Physiol Rep 1:e00173. CrossRef Medline

Baroncelli L, Molinaro A, Cacciante F, Alessandrì MG, Napoli D, Putignano E, Tola J, Leuzzi V, Cioni G, Pizzorusso T (2016) A mouse model for creatine transporter deficiency reveals early onset cognitive impairment and neuropathology associated with brain aging. Hum Mol Genet 25: 4186-4200. CrossRef Medline

Bartels F, Bergel H, Bigalke H, Frevert J, Halpern J, Middlebrook J (1994) Specific antibodies against the $\mathrm{Zn}^{2+}$-binding domain of clostridial neurotoxins restore exocytosis in chromaffin cells treated with tetanus or botulinum A neurotoxin. J Biol Chem 269:8122-8127. Medline

Bomba-Warczak E, Vevea JD, Brittain JM, Figueroa-Bernier A, Tepp WH, Johnson EA, Yeh FL, Chapman ER (2016) Interneuronal transfer and distal action of tetanus toxin and botulinum neurotoxins $\mathrm{A}$ and $\mathrm{D}$ in central neurons HHS public access. Cell Rep 16:1974-1987. CrossRef Medline

Cai BB, Francis J, Brin MF, Broide RS (2017) Botulinum neurotoxin type A-cleaved SNAP25 is confined to primary motor neurons and localized on the plasma membrane following intramuscular toxin injection. Neuroscience 352:155-169. CrossRef Medline

Caleo M, Restani L, Vannini E, Siskova Z, Al-Malki H, Morgan R, O'Connor V, Perry VH (2012) The role of activity in synaptic degeneration in a protein misfolding disease, prion disease. PLoS One 7:e41182. CrossRef Medline

Herreros J, Martí E, Ruiz-Montasell B, Casanova A, Niemann H, Blasi J (1997) Localization of putative receptors for tetanus toxin and botulinum neurotoxin type A in rat central nervous system. Eur J Neurosci 9:2677-2686. CrossRef Medline

Hottinger AF, Azzouz M, Déglon N, Aebischer P, Zurn AD (2000) Complete and long-term rescue of lesioned adult motoneurons by lentiviralmediated expression of glial cell line-derived neurotrophic factor in the facial nucleus. J Neurosci 20:5587-5593. CrossRef Medline

Ippolito DM, Eroglu C (2010) Quantifying synapses: an immunocytochemistrybased assay to quantify synapse number. J Vis Exp 45:2270. CrossRef Medline

Keller JE (2006) Recovery from botulinum neurotoxin poisoning in vivo. Neuroscience 139:629-637. CrossRef Medline

Kutschenko A, Reinert MC, Krez N, Liebetanz D, Rummel A (2017) BoNT/AB hybrid maintains similar duration of paresis as BoNT/A wildtype in murine running wheel assay. Neurotoxicology 59:1-8. CrossRef Medline

Marchand-Pauvert V, Aymard C, Giboin LS, Dominici F, Rossi A, Mazzocchio R (2013) Beyond muscular effects: depression of spinal recurrent inhibition after botulinum neurotoxin A. J Physiol 591:1017-1029. CrossRef Medline

Matak I, Bach-Rojecky L, Filipovic B, Lackovic Z (2011) Behavioral and immunohistochemical evidence for central antinociceptive activity of botulinum toxin A. Neuroscience 186:201-207. CrossRef Medline

Mazzocchio R, Caleo M (2015) More than at the neuromuscular synapse: actions of botulinum neurotoxin $\mathrm{A}$ in the central nervous system. Neuroscientist 21:44-61. CrossRef Medline

Miles GB, Hartley R, Todd AJ, Brownstone RM (2007) Spinal cholinergic interneurons regulate the excitability of motoneurons during locomotion. Proc Natl Acad Sci U S A 104:2448-2453. CrossRef Medline

Moran LB, Graeber MB (2004) The facial nerve axotomy model. Brain Res Brain Res Rev 44:154-178. CrossRef Medline
Morbiato L, Carli L, Johnson EA, Montecucco C, Molgó J, Rossetto O (2007) Neuromuscular paralysis and recovery in mice injected with botulinum neurotoxins A and C. Eur J Neurosci 25:2697-2704. CrossRef Medline

Moreno-López B, de la Cruz RR, Pastor AM, Delgado-García JM (1994) Botulinum neurotoxin alters the discharge characteristics of abducens motoneurons in the alert cat. J Neurophysiol 72:2041-2044. CrossRef Medline

Moreno-López B, Pastor AM, de la Cruz RR, Delgado-García JM (1997) Dose-dependent, central effects of botulinum neurotoxin type A: a pilot study in the alert behaving cat. Neurology 48:456-464. CrossRef Medline

Pastor AM, Moreno-López B, de la Cruz RR, Delgado-García JM (1997) Effects of botulinum neurotoxin type A on abducens motoneurons in the cat: ultrastructural and synaptic alterations. Neuroscience 81:457-478. CrossRef Medline

Pellett S (2012) Learning from the past: historical aspects of bacterial toxins as pharmaceuticals. Curr Opin Microbiol 15:292-299. CrossRef Medline

Pirazzini M, Azarnia Tehran D, Zanetti G, Megighian A, Scorzeto M, Fillo S, Shone CC, Binz T, Rossetto O, Lista F, Montecucco C (2014) Thioredoxin and its reductase are present on synaptic vesicles, and their inhibition prevents the paralysis induced by botulinum neurotoxins. Cell Rep 8:1870-1878. CrossRef Medline

Pirazzini M, Rossetto O, Eleopra R, Montecucco C (2017) Botulinum neurotoxins: biology, pharmacology, and toxicology. Pharmacol Rev 69:200235. CrossRef Medline

Restani L, Antonucci F, Gianfranceschi L, Rossi C, Rossetto O, Caleo M (2011) Evidence for anterograde transport and transcytosis of botulinum neurotoxin A (BoNT/A). J Neurosci 31:15650-15659. CrossRef Medline

Restani L, Giribaldi F, Manich M, Bercsenyi K, Menendez G, Rossetto O, Caleo M, Schiavo G (2012a) Botulinum neurotoxins A and E undergo retrograde axonal transport in primary motor neurons. PLoS Pathog 8:e1003087. CrossRef Medline

Restani L, Novelli E, Bottari D, Leone P, Barone I, Galli-Resta L, Strettoi E, Caleo M (2012b) Botulinum neurotoxin A impairs neurotransmission following retrograde transynaptic transport. Traffic 13:1083-1089. CrossRef Medline

Schindelin J, Arganda-Carreras I, Frise E, Kaynig V, Longair M, Pietzsch T, Preibisch S, Rueden C, Saalfeld S, Schmid B, TinevezJY, White DJ, Hartenstein V, Eliceiri K, Tomancak P, Cardona A (2012) Fiji: an open-source platform for biologicalimage analysis. Nat Methods 9:676-682. CrossRef Medline

Serpe CJ, Kohm AP, Huppenbauer CB, Sanders VM, Jones KJ (1999) Exacerbation of facial motoneuron loss after facial nerve transection in severe combined immunodeficient (scid) mice. J Neurosci 19:RC7. CrossRef Medline

Simpson DM, Blitzer A, Brashear A, Comella C, Dubinsky R, Hallett M, Jankovic J, Karp B, Ludlow CL, Miyasaki JM, Naumann M, So Y (2008) Assessment: botulinum neurotoxin for the treatment of movement disorders (an evidence-based review) report of the Therapeutics and Technology Assessment Subcommittee of the American Academy of Neurology. Neurology 70:1699-1706. CrossRef Medline

Trompetto C, Currà A, Buccolieri A, Suppa A, Abbruzzese G, Berardelli A (2006) Botulinum toxin changes intrafusal feedback in dystonia: a study with the tonic vibration reflex. Mov Disord 21:777-782. CrossRef Medline

Valls-Sole J, Tolosa ES, Ribera G (1991) Neurophysiological observations on the effects of botulinum toxin treatment in patients with dystonic blepharospasm. J Neurol Neurosurg Psychiatry 54:310-313. CrossRef Medline

Vinti M, Costantino F, Bayle N, Simpson DM, Weisz DJ, Gracies JM (2012) Spastic cocontraction in hemiparesis: effects of botulinum toxin. Muscle Nerve 46:926-931. CrossRef Medline

Wang T, Martin S, Papadopulos A, Harper CB, Mavlyutov TA, Niranjan D, Glass NR, Cooper-White JJ, Sibarita JB, Choquet D, Davletov B, Meunier FA (2015) Control of autophagosome axonal retrograde flux by presynaptic activity unveiled using botulinum neurotoxin type A. J Neurosci 35:6179-6194. CrossRef Medline

Wiegand H, Wellhöner HH (1977) The action of botulinum A neurotoxin on the inhibition by antidromic stimulation of the lumbar monosynaptic reflex. Naunyn Schmiedebergs Arch Pharmacol 298:235-238. CrossRef Medline

Wissel J, Bensmail D, Ferreira JJ, Molteni F, Satkunam L, Moraleda S, Rekand T, McGuire J, Scheschonka A, Flatau-Baqué B, Simon O, Rochford ET, Dressler D, Simpson DM (2017) Safety and efficacy of incobotulinumtoxinA doses up to $800 \mathrm{U}$ in limb spasticity: the TOWER study. Neurology 88:1321-1328. CrossRef Medline

Witts EC, Zagoraiou L, Miles GB (2014) Anatomy and function of cholinergic C bouton inputs to motor neurons. J Anat 224:52-60. CrossRef Medline 